\title{
Activités
}

\section{Les tâches appropriées des professeurs d'enseignement professionnel}

Illustration du hiatus entre travail en entreprise et formation

The appropriate tasks of vocational teachers: illustration of the gap between work and training

Las tareas apropiadas de los docentes de las disciplinas profesionales. Ilustración de la distancia que existe entre la formación y el mundo del trabajo

\section{Hélène Veyrac et Nina Asloum}

\section{OpenEdition}

\section{Journals}

Édition électronique

URL : http://journals.openedition.org/activites/2138

DOI : 10.4000/activites. 2138

ISSN : $1765-2723$

Éditeur

ARPACT - Association Recherches et Pratiques sur les ACTivités

\section{Référence électronique}

Hélène Veyrac et Nina Asloum, « Les tâches appropriées des professeurs d'enseignement

professionnel », Activités [En ligne], 6-1 | avril 2009, mis en ligne le 15 avril 2009, consulté le 30 avril 2019. URL : http://journals.openedition.org/activites/2138; DOI : 10.4000/activites.2138

\section{cc)}

Activités est mis à disposition selon les termes de la licence Creative Commons Attribution - Pas d'Utilisation Commerciale - Pas de Modification 4.0 International. 


\title{
Les tâches appropriées des professeurs d'enseignement professionnel
}

\section{Illustration du hiatus entre travail en entreprise et formation}

\author{
Hélène Veyrac \\ Nina Asloum \\ Université de Toulouse \\ Ecole Nationale de Formation Agronomique \\ Toulouse EducAgro: Unité de recherche \\ en didactique des savoirs professionnels, scientifiques et sociaux émergents \\ 2 route de Narbonne BP 22687 \\ 31326 Castanet Tolosan Cedex France \\ helene.veyrac@educagri.fr \\ nina.asloum@educagri.fr
}

\begin{abstract}
The appropriate tasks of vocational teachers: illustration of the gap between work and training.

Teachers of vocational disciplines are faced with particular constraints related to the fact that they teach occupational skills. These skills are described in official reference manuals, which necessarily narrows the field of the practical activity they are intended to cover. This reduction is one of the causes of the gap that separates vocational training and actual professional practice.

To what extent are teachers aware of this gap? What do they do in order to fill it? Answers can be found through the description of "appropriate tasks". Research interviews, lesson observations and self-confrontations with teachers in the domain of landscape planning lead to a better understanding of the work done by teachers in vocational training.

The data we have collected illustrates teachers' different appropriate tasks: accepting the gap and informing the pupils about it, innovative teaching practices, showing the contradictions between reference manuals and effective professional practice. In summary, this paper illustrates how the gap between vocational training and professional practice is filled by the teachers, and contributes to the development of research on the analysis of work by teachers.
\end{abstract}

KEYWORDS

Appropriate task, instructions, integrated courses, vocational teaching, teaching job 


\section{1.- Le travail des enseignants}

L'enseignement fait l'objet de nombreuses recherches, notamment en sciences de l'éducation. Le travail des enseignants fait quant à lui l'objet de recherches récentes mais peu nombreuses. La sociologie de l'éducation contribue à la connaissance du travail des enseignants notamment par un article de Woods en 1977, qui décrit un ensemble de «stratégies de survie» des enseignants, en vue «de rendre compte des montages adaptatifs par lesquels les enseignants "pris au piège » d'un métier de plus en plus ingrat s'efforcent de "sauver les apparences » et d'échapper à la destruction de leur identité. » (Woods, 1977/1997, p. 351). Cet auteur indique que plusieurs innovations (eg. enseignement intégré, enseignement en équipe) menacent l'autonomie des enseignants dans la classe, en hypothéquant les bases de construction de leurs stratégies de survie (fraternisation, domination, négociation, ...). Des travaux plus récents insistent sur la réduction de l'autonomie liée à une complexification des prescriptions et à ses modes d'appropriation par les enseignants (Goigoux, 2002). Tardif et Lessard (1999) analysent le travail des enseignants, en s'appuyant notamment sur des paradigmes ergonomiques. C'est l'activité au quotidien de l'enseignant qui est revisitée. Ils montrent que le travail des enseignants, qui s'inscrit dans un cadre structuré, rigide, est un travail solitaire avec une dimension collective de plus en plus importante. Malgré une organisation très régulée, hiérarchisée, codifiée, très bureaucratisée, ils décrivent l'enseignement comme un « travail flou » et contraint par de multiples prescriptions. Ces contraintes ne sont pas sans conséquences. Un des facteurs de l'augmentation de la souffrance au travail des enseignants réside dans la montée des exigences, «l'évolution de la prescription elle-même est porteuse de ces nouvelles exigences et semble infaisable». Le métier d'enseignant y est alors parfois qualifié de «métier impossible ( (Lantheaume, \& Hélou, 2008, p. 35). La liberté pédagogique semble mise à mal par une complexification des contraintes et un éclatement du sens de l'institution scolaire. L'école fait l'objet d'attentes multiples, parfois incompatibles. Les injonctions paradoxales obligent les enseignants à faire des choix, des arbitrages, et à les assumer. Ils ne peuvent guère satisfaire l'ensemble des «publics-prescripteurs » que sont notamment les apprenants et les parents, qui ont des exigences hétérogènes voire divergentes vis-àvis d'une activité multi-adressée.

Pour Amigues (2002), les recherches sur l'appropriation des prescriptions par les enseignants et sur le rôle des prescriptions dans le système enseignement-apprentissage, font l'objet de «zones d'ombre dans la recherche en éducation» (ibid., p. 200). Depuis, des recherches sur ce sujet émergent (Goigoux, 2002) ainsi que des recherches en ergonomie (eg. Rogalski, 2003; Litim, Prot, Roger, Ruelland, \& Clot, 2005; Leblanc, Ria, Dieumegard, Serres, \& Durrand, 2008). Par ailleurs, des recherches épidémiologiques sur le «burn out enseignant » et sur les inconvénients redoutés par les enseignants (sentiments d'impuissance, agressions verbales, responsabilité morale qui incombe au métier, etc.) apparaissent (eg. Kovess-Masféty, Seidel, \& Sévilla, 2006). Elles montrent la nécessité d'études qualitatives pour éclairer les liens entre les facteurs de risque qu'elles mettent statistiquement en évidence et le sens du travail.

L'ensemble de ces recherches ont un champ de validité varié: elles éclairent soit le travail de tous les enseignants d'un pays, soit celui d'enseignants de matières «générales » d'un pays (français, philosophie, mathématiques), soit encore celui de professeurs d'écoles élémentaires d'un pays. Concernant les enseignants de matières professionnelles, les conditions de travail sont spécifiques, de même qu'une partie de leurs difficultés (Veyrac, \& Chatigny, 2007). Les prescriptions qui leur sont imposées sont particulières, notamment du fait de la nature des savoirs à enseigner. Ces savoirs sont énoncés dans les référentiels de formation. Ils constituent une prescription basée sur des analyses de pratiques professionnelles. La formalisation et la mise en texte de pratiques professionnelles, la transformation de référentiels professionnels en référentiels de formation opèrent une réduction de l'activité professionnelle visée par les formations (Raisky, 1993; Bouillier, 2006; Rey, 2006). Ainsi apparaissent des écarts, des hiatus entre ce qui est prescrit d'enseigner dans les référentiels et les pratiques professionnelles effectives. On parlera dans cet article de hiatus entre travail en entreprise et formation pour désigner ces écarts. 
Ce hiatus est illustré par Delgoulet (2001), à propos d'un échange entre un stagiaire et un formateur lors d'une formation dans une entreprise française de transport ferroviaire. Le savoir à enseigner visé par le formateur est «qu'une pièce doit être totalement propre (sans calamine) pour que l'on puisse faire un examen par magnétoscopie » (ibid, p. 5).

Stagiaire: « Mais on le fait en pratique » [la magnétoscopie d'une pièce faiblement calaminée]

Formateur: «Oui, mais en théorie on ne doit pas le faire.»

Stagiaire: «Oui, mais nous on le fait. Nos «X $»$ [pièces de moteur], ils sont toujours calaminés. »

Formateur: «Il n'y a pas moyen de les nettoyer? »

Stagiaire: «Non»

Formateur: « Je ne sais pas moi, je les connais pas tes pièces. [...]

Ainsi est illustré le sentiment de fort décalage entre ce qui est enseigné en établissement de formation professionnelle et ce qui est pratiqué par les professionnels.

Delgoulet (ibid.,p.11) note que « ces controverses [entre les pratiques enseignées et celles au travail] interrogent [...] le niveau de compétences pédagogiques et techniques nécessaires au formateur dans la situation, son rôle et ses marges d'action face à un enseignement ici très standardisé.» L'échange montre en effet la gêne du formateur qui s'excuse de son ignorance ( « je ne sais pas moi, je les connais pas tes pièces »), probablement pour tenter de clore la controverse sur le hiatus.

Quelles marges de manœuvre les enseignants de formation professionnelle s'accordent-ils vis-à-vis de ces controverses? Perçoivent-ils des écarts entre ce qu'ils enseignent et ce que les professionnels pratiquent? Quel sens donnent-ils à ces écarts ? Cherchent-ils à les réduire?

Pour apporter des éléments de réponse, le concept de «tâche appropriée » (cf. Falzon, 2004) est ici convoqué. Il s'agit de rendre compte des buts que l'agent se fixe pour lui-même, des orientations qu'il donne à son action et ainsi de contribuer à décrire le travail des agents de leurs points de vue. Le concept de tâche appropriée permet d'approcher ce que les agents se donnent à faire, de manière dépendante ou non de ce qu'ils pensent prescrit. Il ne s'agit pas ici d'évoquer ce que l'agent comprend de la tâche prescrite. Les concepts de «tâche comprise » (e.g. Falzon, 2004) ou de «tâche prescrite pour l'agent » (Leplat, 1997) ne seront donc pas utilisés. L'étude exploratoire présentée dans cet article vise à exposer les points de vue d'enseignants sur une part du sens de leur travail constitué de ce qu'ils se donnent à enseigner, indépendamment de l'analyse de ce qu'ils pensent qu'on leur prescrit.

\section{2.- Méthodologie}

\section{1.- Contexte de l'étude}

Les données recueillies sont issues d'une recherche-action sur les difficultés des enseignants à intégrer l'interdisciplinarité dans leur pratique quotidienne (Bouillier-Oudot, \& Asloum, 2004) et sur le travail collectif imposé par les formations interdisciplinaires (Asloum, \& Bouillier-Oudot, 2007). Cette recherche-action s'est déroulée sur deux années avec des équipes d'enseignants de cinq établissements préparant au Brevet de Technicien Supérieur Agricole (BTSA) en aménagement paysager. Ce diplôme, délivré par le ministère de l'agriculture français, est préparé en deux années après le baccalauréat. Les enseignants ont principalement participé à des groupes de réflexion sur la mise en place de situations interdisciplinaires. Deux de ces cinq établissements ont été retenus pour la phase de suivi de la recherche-action, sur le critère du volontariat. La présente étude s'appuie sur les données recueillies lors de ce suivi. 


\section{2.- Premier établissement}

\section{Recueil}

Un entretien semi-directif venait clore la recherche-action; il visait à recueillir les avis des enseignants sur leurs pratiques pluridisciplinaires et laissait une large part à la prise en compte des points de vue des enseignants sur leur travail. C'est dans ce climat de confiance que deux enseignants, coordonnateurs de la filière, ont abordé librement leurs difficultés, qui se centraient sur le hiatus école/ entreprise. L'entretien de plus de deux heures a mis en présence ces deux enseignants d'un même établissement du nord de la France, dans la même discipline, préparant au même diplôme. Monsieur $\mathrm{B}$ avait en charge une classe d'étudiants en formation initiale, alors que Madame V avait en charge une classe par alternance (Centre de Formation par l'Apprentissage). Monsieur B avait une longue expérience de l'enseignement et une expérience professionnelle du métier de paysagiste alors que Madame V était une jeune enseignante débutante avec une courte expérience professionnelle dans l'aménagement paysager.

\section{Traitement des données}

L'entretien a été enregistré et retranscrit. Une analyse sémantique a permis de constituer un corpus réduit aux énoncés portant sur le thème du hiatus formation/travail. Ce corpus réduit a fait l'objet d'une analyse de contenu (Bardin, 1977) qui a permis de dégager des catégories correspondantes aux questions suivantes. Est-ce que les enseignants constatent un hiatus entre les pratiques professionnelles qu'ils enseignent et celles qu'ils imaginent être les pratiques dominantes des professionnels? Si oui, à quoi attribuent-ils ce hiatus, quelles en sont les causes selon eux? Quelles sont les incidences de ce hiatus?

\section{3.- Second établissement}

Comme dans le cas du premier établissement, c'est lors d'une rencontre organisée dans la dernière phase de suivi de notre recherche sur l'interdisciplinarité que deux enseignants ont été interviewés. L'entretien a duré deux heures. Un des deux nous a indiqué qu'après des années de difficultés à exploiter les séances de retour de stage - moment charnière de l'alternance - il y parvenait de manière satisfaisante. Il nous a alors sollicités pour que nous le filmions lors de ces séances de retour de stage de ses élèves. Bien que la demande vienne de lui, nous avons pris les précautions déontologiques d'usage. Les étudiants et lui ont été assurés que les informations recueillies seraient utilisées exclusivement à des fins de recherche ou de formation. Les objectifs de cette recherche ont été explicités aux participants qui ont complété et signé un formulaire cédant leur droit à l'image.

L'enseignant en aménagement paysager travaille depuis plus de quinze ans dans un centre de formation par alternance pour apprentis en formation initiale préparant le BTSA en aménagement paysager. Il a longuement exercé dans le domaine de l'aménagement paysager en tant que professionnel avant d'intégrer par la suite l'enseignement agricole public. Cet enseignant a par ailleurs suivi une formation relative à l'analyse de pratiques pédagogiques.

\section{Recueil des données}

Les données ont été recueillies par entretien, observation et auto-confrontation.

- Comme pour le premier établissement, un entretien semi-directif, mené par les mêmes chercheurs, a été enregistré et retranscrit.

- Ensuite, une séance d'enseignement de retour de stage a été filmée. Une mini-caméra cravate, dite caméra « subjective », a été fixée à hauteur de poitrine de l'enseignant, avec un angle de prise de vue se rapprochant de la vision humaine. Elle enregistrerait les prises d'informations visuelles et auditives de l'enseignant. Cette caméra subjective, utilisée dans des recherches en ergonomie pour l'étude des usages d'artefacts mobiles (Salembier, Kia, Dieumegard, Serres, 
\& Durrand, 2005), a permis de ne pas confronter l'enseignant à sa propre image, dans le but d'éviter ainsi une situation d'autoscopie lors de l'auto-confrontation. Nous avons également positionné deux caméras, à deux angles opposés de la salle: une première en fond de salle cadrant le tableau et l'enseignant et une deuxième côté tableau, cadrant les étudiants, au moyen de l'action d'un cameraman.

- L'auto-confrontation a été filmée. La caméra enregistrait la télévision sur laquelle était projeté le film issu de la caméra subjective et les commentaires de l'enseignant. L'auto-confrontation s'est déroulée consécutivement aux deux heures d'enseignement. L'enseignant interrompait le déroulement du film lorsqu'il le souhaitait et le commentait. Les commentaires ne portent donc pas sur tous les temps de la séance d'enseignement.

\section{Traitement des données}

Les données verbales de la séance d'enseignement et de l'auto-confrontation ont été entièrement retranscrites. Nous avons alors retenu les données verbales portant sur les situations professionnelles relatées par les étudiants où les écarts entre pratiques du référentiel et pratiques effectives étaient les plus fortes, afin de mieux illustrer la tâche appropriée relative au hiatus formation / travail en entreprise. Les éventuels commentaires de l'enseignant relevés au cours de l'auto-confrontation et relatifs à ces situations professionnelles complètent le corpus.

\section{3.- Le hiatus formation/entreprise : du constat à la tâche appropriée}

Les données présentées ci-après ont été recueillies dans le premier établissement.

\section{1.- Constat d'un hiatus par les enseignants}

Les enseignants du premier établissement font le constat de hiatus formation/entreprise notamment à propos de l'étude paysagère ${ }^{1}$. Les deux enseignants s'accordent sur le fait que l'étude paysagère n'est pas formellement appliquée par les professionnels, elle est menée très succinctement.

B (Monsieur B): Parfois tu sais, on voit des paysagistes dans le coin, l'analyse du site: deux trois photos et puis vas-y; et encore quand il y a des photos! Parfois ils font des projets d'aménagement, ils ne connaissent pas le site.

On a vu des grands paysagistes du coin [ne pas faire d'étude paysagère] [...].

On arrive à des choses qui sont quand même folles : je me rappelle d'un travail qui a été fait, thématique aussi, sur l'intégration de fermes dans la région, dans les Flandres, et donc on est dans des openfields etc., et donc on se retrouvait avec des gens - il y avait plusieurs écoles locales qui travaillaient là-dessus - et on se retrouvait avec des gens qui enfermaient complètement la ferme; c'est-à-dire que finalement, ils ne faisaient pas d'étude du paysage, et l'absence de cette étude du paysage les ramenait à faire des choses complètement aberrantes; ou à implanter un élément, un élément [choquant] en plein milieu de la campagne, tu vois ce genre de truc, ça passe pas quand même inaperçu.

Le constat d'écarts entre ce qui est enseigné et ce qui est pratiqué consiste ici, de la part de Monsieur $\mathrm{B}$, à porter un jugement négatif sur certaines pratiques professionnelles. Mais cet enseignant développe son analyse et envisage diverses causes d'écarts.

1. Le référentiel de formation précise que les enseignants doivent former les élèves et apprentis aux différentes étapes du métier de technicien paysagiste. Un des objectifs généraux de la formation, celui de l'étude paysagère, est inscrit ainsi dans le référentiel: «Etre capable de maîtriser les techniques utiles à l'étude paysagère préalable à un aménagement ». Cinq étapes sont précisées pour mener une étude paysagère:

«I - Analyser les objectifs et les contenus d'une étude préalable

I I - Utiliser les documents nécessaires à la réalisation d'une étude préalable

III - Effectuer une étude de site dans le cadre d'une lecture de paysage

IV - Effectuer des relevés sur le terrain

V - Proposer un diagnostic concernant le site». 


\section{2- Attribution des causes du hiatus par les enseignants}

Les extraits d'entretien suivants illustrent quatre types d'attributions causales, par les enseignants, des décalages entre référentiels de formation et pratiques professionnelles. Ces quatre types ont été définis par une analyse de contenu.

\section{Les procédures atomisées du débutant vs. les procédures automatisées de l'expert}

B : tu sais, toi tu es paysagiste, quand tu es paysagiste, tu as une expérience de terrain etc., et quand tu vas sur un site, et bien tout de suite tu as le regard.

Tu prends pas forcément la peine de tout noter, tu as des choses qui te restent, tu prends des petites notes très très rapides.

L'analyse du paysage est, pour cet enseignant, une étape de la démarche professionnelle qui s'automatise avec l'expérience et devient non consciente. La procédure enseignée est " atomisée », au sens de Vermersch (1985): elle précise toutes les étapes, dans un niveau de détail élevé. L'expérience provoque une automatisation progressive de cette procédure, une "procéduralisation », qui est un processus qui a fait l'objet de nombreuses descriptions en psychologie cognitive (eg Anderson, \& Fincham, 1994). Le décalage entre pratiques professionnelles effectives et pratiques enseignées serait ici inhérent au processus d'apprentissage: il ne s'agirait pas d'une étape rejetée par les professionnels mais d'une étape compilée.

\section{Étendue du domaine d'application des pratiques}

B : «Ils [les professionnels] vont le faire [analyse du paysage] quand ils sont jeunes parce qu'ils connaissent pas forcément très bien leur région, mais très rapidement ils savent comment va se présenter le sol à tel endroit; il a une connaissance de son territoire ».

[...] L'étude du sol: quand tu es un professionnel de la région, tu sais que dans le Nord [région française] ce sera plutôt limono-argileux, là ce sera plutôt argileux, etc. Tu as déjà ces connaissances là donc tu n'as pas besoin de les écrire puisque tu connais ton site.

Et finalement, l'étudiant, quand il se retrouve dans un cadre d'une entreprise dont les gens ont déjà ces compétences-là, et bien ça passe d'une manière vraiment anodine par rapport aux étudiants.

Les techniciens paysagistes en exercice interviennent souvent sur un espace géographique relativement réduit. Les référentiels de formation visent à former des professionnels aptes à caractériser un ensemble de sols important, ils visent à permettre aux futurs professionnels de pratiquer dans des situations bien plus diversifiées que celles d'un professionnel expérimenté.

Les propos de Madame V illustrent encore cette cause d'écart entre pratiques effectives et pratiques enseignées.

V (Madame V) « Donc eux [les professionnels], ils font simplement le côté accessibilité au chantier, le côté purement pratique; en plus, pour peu que ce soit un appel d'offre [...] eux, dans l'appel d'offre, qu'est-ce qu'on leur dit? On leur dit d'appliquer le cahier des charges, donc ils font pas trop de recherches autres ».

Pour cette enseignante, le référentiel de formation couvre plus de techniques que les pratiques professionnelles effectives. L'étude paysagère est bien souvent effectuée en amont, par des bureaux d'étude, et ne relève pas toujours de l'activité du technicien. On perçoit ici un écart potentiel entre le champ étendu des pratiques professionnelles du référentiel de formation et les pratiques professionnelles effectives que se représente cette enseignante. Le champ d'application du référentiel serait plus vaste que les responsabilités effectivement assumées par les professionnels et serait ainsi une cause du hiatus formation et entreprise.

Il s'agit ici d'une réduction subie de l'étendue du domaine d'application, contrairement à l'illustration précédente qui renvoie à une réduction plus « maîtrisée » due à l'expérience professionnelle. 


\section{Contraintes de rentabilité}

Les enseignants évoquent les contraintes de rentabilité auxquelles sont soumis les professionnels et non les étudiants.

B : Et comme dans la région ils font aussi beaucoup de petits chantiers, [...] et bien finalement sur un petit chantier, tu vas quand même pas prendre le temps [quand tu es professionnel], si il [le chantier] fait dans [les] 2000 ou $3000 \mathrm{~m}^{2}$, tu vas pas prendre le temps de faire une analyse complète par rapport à ça.

Sur 5 à 8 projets que va faire un paysagiste, ben il va peut-être en avoir 2 [qui feront l'objet d'un contrat]; donc financièrement [c'est pas rentable d'y passer trop de temps]; et puis bon parfois, on lui demande une estimation, donc ça se fait à l'arrache, il est débordé de boulot et finalement [...] en fonction du client, en fonction de l'importance de l'enveloppe qu'il peut recevoir [...]. [...] on voudrait pas généraliser non plus : tous les professionnels ne bâclent pas leur travail.

V: Je pense qu'ils [les professionnels] n'ont pas le temps de faire une analyse aussi poussée de tout l'environnement lorsqu'ils abordent un chantier.

Les contraintes de rentabilité sont, aux yeux de ces deux professeurs, des contraintes si pesantes que l'étape d'analyse du paysage, coûteuse en temps, serait négligée par les professionnels. La dimension constructive de l'activité, au sens de Samurçay et Rabardel (2004), serait privilégiée par la formation. Pour ces enseignants, le fait de ne pas prendre en compte la dimension marchande des pratiques professionnelles serait un facteur du hiatus.

Cet aspect déjà décrit (e.g. Degoulet, 2001) semble récurrent dans les formations professionnelles, comme si les instituts de formation avaient en charge les aspects « constructifs » de l'activité et l'entreprise les aspects «productifs », à charge des apprenants de faire avec cette tension. Pourtant, les référentiels de formation n'évacuent pas strictement la dimension productive puisqu'ils l'abordent notamment par des enseignements en économie. Plusieurs hypothèses peuvent être avancées: (1) le morcellement des savoirs opéré par les référentiels de formation conduit à réserver la contrainte financière à quelques parties d'enseignement, (2) les enseignants résistent à un enjeu de leur fonction qui est de préparer les apprenants à participer à l'appareil de production capitaliste, (3) les apprentissages nécessitent du temps et ne sont par nature pas rentables, (4) les contraintes financières conduisent à des pratiques professionnelles peu avouables qu'on ne peut déontologiquement guère enseigner.

\section{Prise en compte de l'évolution technico-scientifique et des demandes sociales}

Le hiatus est également attribué aux évolutions scientifiques et techniques. Par exemple, de nouvelles pratiques sont prônées par le développement durable, mais encore peu développées par les professionnels.

B: Le non tuteurage, par exemple, le non bêchage, c'est des problèmes d'actualité, les plantations en bute, c'est des problèmes d'actualité, le drainage que l'on fait avec les tubes à l'intérieur, avec les drains [...] ça devrait plus se faire; c'est tout ce genre de choses qui crée des décalages entre le discours que je leur présente puis le discours de l'examen; et on l'invente pas.

Monsieur B cite ainsi des pratiques qui se développent notamment du fait de la demande sociale en matière de respect de l'environnement. Précisons que cet enseignant milite pour la taille-douce des arbres dans des organisations regroupant professionnels et chercheurs et dispose ainsi, sur le thème de la taille-douce, des références à des pratiques nouvelles très peu développées qui n'apparaissent pas encore dans les référentiels de formation. Un triple hiatus apparait ici: prescription de formation/formation effective/entreprise, qui posent potentiellement des problèmes aux étudiants lors des épreuves d'examen où l'évaluation échappe à l'enseignant. 


\section{3.- Incidence du hiatus pour les enseignants}

Le hiatus est source de difficulté, comme le précisent les données suivantes.
$\mathrm{V}$ : Nous [enseignants] ça [la conscience qu'ont les étudiants des écarts entre pratiques enseignées et pratiques effectives des professionnels] nous freine dans l'apprentissage parce qu'ils [les étudiants] se disent « ben ça on s'en fout » «de toutes façons, on le fait jamais en entreprise », « à quoi ça sert », « à quoi ça sert de faire toutes ces études là, nous dans l'entreprise on est là pour planter des choux » [exécuter des ordres simples] donc du coup ça nous freine, parce que comme ils en voient pas l'utilité, du coup ils s'investissent pas.
B : On [les professionnels] leur aura bassiné pendant des années en leur disant « il faut toujours tailler après la floraison », $[\ldots]$ nous on peut prouver maintenant qu'il y a des tailles qui se font, différentes, au niveau des arbustes par exemple.
Finalement, c'est pas ce que fait le professionnel.
$\mathrm{V}$ : C'est clair qu'on est en décalage.
B : Au niveau de la taille des arbustes, par exemple, on est en décalage total.
$\mathrm{C}$ (Chercheur): Et ça ils [les étudiants] le soulèvent cet aspect là.
B: Oh ben bien sûr.
$\mathrm{V}$ : On le soulève avant eux, moi c'est la première chose que je leur dis.
B: Il y a des techniques qui ont quand même 10,15 ans que ne devraient plus se faire et qui sont encore dans les manuels professionnels et puis quand on corrige les copies au mois de juin, on voit quand même le retard de certains enseignants dans le domaine, c'est quand même un peu triste parfois [ces copies du mois de juin sont des copies d'étudiants d'autres établissements de formation.]

Cet échange illustre plusieurs ressentis relatifs à l'isolement des enseignants: le sentiment d'enseigner des contenus inadaptés aux attentes exprimées par les étudiants, le sentiment d'enseigner à contre-courant des pratiques professionnelles dominantes, le sentiment d'enseigner à contre-courant de collègues enseignant dans des établissements différents.

\section{4.- Les tâches appropriées des enseignants}

Les précédentes données définissent une multiplicité d'attributions causales des hiatus formation/ entreprise. Selon les enseignants, les hiatus peuvent provenir, (1) de la rapidité de réalisation des étapes dues à l'expertise des professionnels, (2) de la différence d'étendue du domaine d'application des pratiques professionnelles et des pratiques enseignées, (3) de la faible prise en compte des contraintes de rentabilité par les formateurs, ou encore (4) des évolutions scientifiques et techniques. Les discours analysés laissent apparaître un hiatus inéluctable, intrinsèque au travail d'enseignant en formation professionnelle. Les enseignants, qui semblent en accord sur le constat du hiatus et sur ses causes, se donnent-ils pour autant les mêmes savoirs à enseigner? Les données tendent à affirmer que non. En effet, deux tâches appropriées se dégagent.

- Le constat d'écarts entre pratiques effectives et pratiques enseignées est posé d'emblée par Madame V: «c'est la première chose que je leur dis ». Tout se passe comme si le décalage était un fait établit, sans alternative possible. La richesse des attributions causales à ce hiatus accentue son caractère inéluctable. Des « décalages » existent donc, qu'elle annonce assumer en les posant aux élèves comme un fait non discutable. On notera qu'elle évoque des difficultés d'ordre motivationnel: «ils ne voient pas l'utilité », «ils ne s'investissent pas », qui s'associent probablement de difficultés dans la conduite de la classe.

La tâche appropriée de cette enseignante s'appuie sur la prescription. On sait que les enseignants plus expérimentés s'autorisent des choix et s'accordent plus de temps à la gestion des relations pédagogiques dans l'interaction avec les apprenants (Tochon, 1993). Par ailleurs, ces résultats sont en accord avec les travaux de Caroly et Weill-Fassina (2004) qui montrent, dans une relation de service (guichetier-clients de services postaux), que les agents jeunes et peu expérimentés appliquent les consignes, pour éviter de se mettre en conflit avec leur hiérarchie. Ce faisant, ils adoptent des stra- 
tégies pour concilier le client en lui donnant des explications sur les prescriptions, comme pour «se dédouaner ».

- Monsieur B, regrette quant à lui l'obsolescence de certains enseignements qu'il qualifie de «en décalage », comme le tuteurage, le bêchage. Comme Madame V, il comprend et explique les hiatus. Pourtant, il semble quant à lui se donner à enseigner des pratiques professionnelles qui ne sont ni celles du référentiel, ni les pratiques effectives dominantes, mais des pratiques effectives émergentes. Il s'agit de pratiques novatrices, «d'actualité », peu développées. Notons que cette tâche appropriée ne réduit pas nécessairement le hiatus entre institut de formation et entreprise. En effet, en prenant comme référence à enseigner des pratiques professionnelles novatrices, l'institut de formation se positionne davantage comme une « vitrine », promouvant des pratiques souhaitables peu développées, que comme un «miroir» des pratiques professionnelles dominantes. Par ailleurs, les écarts entre référentiel de formation et formation effective créent des tensions pour les étudiants, notamment lors des évaluations de l'apprentissage menées par des tiers, qu'ils soient enseignants ou professionnels.

Les deux tâches appropriées de professeurs, comme elles viennent d'être décrites, ne réduisent guère les hiatus formation/travail. Une troisième tâche, plus réductrice des hiatus va maintenant être illustrée.

\section{4.- Réduire le hiatus en provoquant un discours sur les pratiques effectives}

L'enseignant du deuxième établissement organise des séances de « restitution de phase » à chaque retour de stage des étudiants au centre de formation. Ces séances sont consacrées à l'explicitation par les étudiants des expériences vécues en entreprise. Le professeur exprime le sens qu'il donne à ces séances ainsi : "On ne peut pas ignorer que les jeunes ont passé un mois en entreprise [...] on a une phase où ils vont nous restituer à l'oral et où ils vont nous restituer des documents par écrit: cette phase-là est essentielle parce que c'est un moyen de raccorder "voilà ce que j'ai vécu, voici ce qu'on va en faire ici » [...] c'est un moment qui pour nous est essentiel parce que c'est presque un moment interdisciplinaire où on relie ce qu'ils font en pratique, on relie la théorie, on relie l'expertise ». En mettant en place ces séances, ce professeur « cherche à recueillir le vécu des étudiants en entreprise, évaluer le niveau d'autonomie de chaque étudiant, évaluer le niveau de conceptualisation de chaque étudiant, évaluer le potentiel de l'entreprise à participer à la formation du jeune, évaluer si l'étudiant utilise l'entreprise comme une structure de formation, mieux connaître les étudiants, identifier des besoins collectifs de formation, se tenir informé des évolutions de la profession d'aménagement paysager » (Bouillier, Asloum, \& Veyrac, 2008, p. 202).

L'enregistrement audiovisuel d'une séance de retour de stage de début d'année et la retranscription des auto confrontations nous fournissent plusieurs exemples où les pratiques professionnelles effectives sont évoquées par les étudiants alors qu'elles ne correspondent pas aux pratiques du référentiel étant donné leur caractère déconseillé voire dangereux, braconnier et clandestin. Quatre de ces pratiques sont présentées ci-après. Elles concernent le non-respect d'une procédure lors d'un appel d'offres, le port du casque sur les chantiers, l'enfouissement de déchets à l'insu de clients et le nonrespect des délais de séchage du ciment.

\section{1.- L'appel d'offres: permettre le récit de pratiques illégales}

Au cours de la séance de retour de stage, chaque étudiant a un temps de parole d'une dizaine de minutes pour présenter à la classe entière une pratique professionnelle vécue ou observée. Ici, une étudiante met en débat une pratique qui lui semble illégale.

E1 (Etudiante 1): Nous on a eu un souci avec un devis d'appel d'offres, parce qu'il [le maître d'apprentissage] voulait faire une plantation d'alignement d'Albizia julibrissin [...] pour un devis, il nous fallait au moins trois entreprises [...] on pouvait pas en fait avoir ces trois pépinières pour 
faire un devis.

[l'étudiant explique le montage qui a été effectué par son entreprise].

Etudiant 2: Il n'y a pas trop eu de mise en concurrence là.

E1 : Mais non, il n'y en a pas eu.

$\mathrm{P}$ (Professeur): On peut faire un appel d'offres international, mais dans certains cas peut-être que

c'est prévu dans la loi, ça.

Etudiant 3 : Pourquoi, puisque pour la loi c'est pas terrible ça; normalement, il faut qu'il y ait forcément mise en concurrence.

P: Mais c'est pas forcément; on peut avoir l'exclusivité sur un matériel; c'est possible ça sur certain matériel.

$[\ldots]$

P: Il y a peut-être des textes de loi là-dessus; ce serait intéressant de se pencher là dessus et de demander l'avis de [Nom d'un maître d'apprentissage d'un autre étudiant] là-dessus : parce que tu as un spécialiste avec toi, d'appel d'offres, de lui demander le cas de quelqu'un comme ça pour une série d'arbres [...].

La partie qui suit est l'extrait de l'auto-confrontation qui concerne l'échange précédent: P commente l'extrait de film correspondant à la retranscription ci-dessus.

P: Elle [l'étudiante E1] parle d'un cas, d'un montage, en fait; elle cherche à savoir si ce qui se fait [dans son lieu de stage] est juste [...] Ils n'ont pas fait d'appel d'offres [...].

En fait le problème c'est que elle, elle n'a pas la réponse, elle ne sait pas où aller pour avoir la réponse.

Le fait qu'elle le mette en pâture, c'est bien pour qu'on en débatte, qu'on en parle.

Mais encore une fois, le cours sur l'appel d'offre, c'est à Noël de l'année prochaine [qu'il est programmé].

$\mathrm{C}$ (Chercheur) : à ce moment là, tu l'as la réponse [à la question de l'étudiante.

P: [...] Ben non là je l'ai pas, de toute façon, je crois qu'un enseignant en apprentissage est perpétuellement sur la sellette [...] au tout début quand je suis arrivé au CFA, je voulais à tout prix chercher la réponse.

L'enseignant ne porte pas de jugement sur le caractère illégal de l'appel d'offres, son discours en classe est à visée compréhensive, ce qui permet à l'étudiante de relater les pratiques effectives. L'auto-confrontation dévoile la tâche appropriée : l'enseignant ne se donne (plus) comme mission de « chercher à tout prix la réponse » à une question soulevée par un étudiant. En effet, dans l'extrait, il demande à un étudiant (différent de l'étudiant qui rend compte de son expérience) de se renseigner sur les textes de lois qui régissent les appels d'offre. Il ne s'engage pas à répondre sur ce point, mais renvoie la question, et incite les étudiants à «faire circuler » les savoirs professionnels entre eux et leurs maîtres d'apprentissage. La tâche appropriée est alors de permettre des débats pour favoriser l'acquisition de savoirs.

\section{2.- Le port du casque : chercher à dévoiler des pratiques dangereuses}

Un autre étudiant évoque un changement intervenu dans le port d'équipement de protection individuelle.

E4 (Etudiant 4): Depuis cette semaine-là, quand on travaille avec la mini-pelle, on est obligé de mettre le casque.

$\mathrm{P}$ : Ah, bon: mais pourquoi depuis cette semaine?

E4 : Parce qu'il y a un accident, enfin, qui n'a rien à voir avec ça mais ; on a un stagiaire qui s'est pris une branche sur la tête, il aurait pu y passer; depuis ce temps-là le chef il veut qu'on fasse plus attention à ça.

$[\ldots]$

$\mathrm{P}$ : et oui oui oui, « casque obligatoire !» quand il y a une machine qui tourne autour là ; c'est embêtant ça! [c'est embêtant de porter un casque].

E4: Oh, ça fait un style.

Les autres étudiants : Rire 
$[\ldots]$

P: ça fait un style oui, ça c'est sûr.

E4: J'ai fait un peu de mini-pelle aussi.

P: C'est pour ça que vous aviez mis le casque [sous-entendu: tu étais dangereux pour les autres].

Les autres étudiants : Rire

La formation à la santé sécurité au travail fait partie des référentiels du diplôme préparé par les étudiants. L'enseignant, en utilisant l'humour, montre à l'ensemble des étudiants qu'il entend que les pratiques effectives ne sont pas toujours conformes aux réglementations. Il semble tenter («c'est embêtant ça»), en vain, à faire reconnaître à l'étudiant qu'il ne portait pas d'équipement de protection individuelle.

\section{3.- L'enterrement civil : déculpabiliser le récit de pratiques inélégantes}

Un étudiant a participé à un chantier pour lequel il a aménagé des terrains pour les engazonner. Cette pratique professionnelle est génératrice de déchets. L'enseignant l'interpelle alors sur ce sujet.

P: Qu'est-ce que vous faites des déchets? quand on fait des gazons, il y a beaucoup de déchets: des cailloux, des...

[l'étudiant explique qu'une machine enlève les petits déchets]

P: Est-ce que vous faites des trous et vous mettez tout dedans?

E5 (Etudiant 6): Ben, c'est « l'enterrement civil » qu'ils appellent ça [RIRE]

$\mathrm{P}:$ L'enterrement?

E5 : «L'enterrement civil », ils appellent ça: un petit trou et puis, quatre brouettes de cailloux.

P: C'est assez fréquent dans les entreprises, c'est pour ça que je t'en parle.

E5 : Ouais de toutes façons, c'est obligatoire, ça évite de le monter dans le camion, pour le client aussi ça fait moins cher; on le laisse chez lui, caché.

Une majorité d'étudiants: Rires

E6: « Surprise !» quand il [le client] creuse.

P: Tu sais combien ça coûte [d'évacuer les déchets]?

E5 : Je sais pas exactement mais ça doit revenir quand même assez cher.

P: Quelqu'un a une idée du prix?

E7 (Etudiant 7): Ben ça dépend si on les laisse, si on les met en dépôt ou

P: Ouais mais de toute façon, il [le professionnel] va s'en débarrasser quand même des caillasses et tout.

E7 : Oui mais si il y a une décharge qui les prend dans l'entreprise, là ça coûte rien, [dans] tous les cas, le camion, il faut bien qu'il le ramène à l'entreprise, et si on fait une décharge dans l'entreprise.

P: Ouais, ça coûte rien, d'accord.

Dans cet échange, il est question d'une pratique peu avouable: les déchets issus des travaux (gravats, cailloux, végétaux...) sont enfouis sous-terre. À l'insu des clients, les professionnels dissimulent les déchets. Leur évacuation est potentiellement coûteuse en temps, en matériel et pénible pour les agents. De plus, si l'entreprise ne dispose pas elle-même d'un système de déchetterie, des frais de déchetteries lui sont facturés, bien souvent à des tarifs relativement élevés.

L'enseignant connaît cette pratique : l'analyse de l'échange révèle la volonté de l'enseignant d'aborder ce sujet: "Qu'est-ce que vous faites des déchets? 》 puis «Est-ce que vous faites des trous et vous mettez tout dedans? ». Soulignons que cette pratique est institutionnalisée au point d'être désignée par un terme stabilisé appartenant probablement au langage opératif du groupe de travail de l'apprenti.

Voici l'extrait de l'entretien d'auto confrontation correspondant à cette pratique.

P: Quand je lui parlais de cette histoire de déchets, c'est amusant parce que j'ai l'impression que j'agressais son entreprise: en disant « mais qu'est-ce vous faites des déchets ». Alors il a avoué:

« ben nous on fait un enterrement civil ». Euh, et là ce qui est intéressant c'est de, c'est assez étonnant de, qu'il se sente en porte-à-faux, et c'est pour ça que j'ai senti le besoin de lui dire que 
toutes les entreprises le font, pour éviter qu'il euh

C: Pour éviter qu'il dénigre son entreprise

$\mathrm{P}$ : Oui parce qu'il, on se sent toujours en porte-à-faux c'est-à-dire, il se dit « je dénonce quelque chose ». La jeune fille, un peu plus tard, qui a dit « écoutez j'ai été obligée de faire un appel d'offres on a dû bidouiller quelque chose parce qu'on était pas très sûr ». Bon je crois que c'est vrai que c'est bien d'en parler, c'est vrai que c'est bien pour moi de le redire avant qu'ils vont partir du CFA, je vais le redire « attention tout ça c'est entre nous, vous n'avez pas à divulguer quoi que ce soit qui se discute ici ». Néanmoins, ce serait l'occasion de se renseigner: toutes les entreprises enterrent leurs déchets, toutes les entreprises quand on a un seul fournisseur, on est obligé de...[ne pas respecter les procédures d'appels d'offres] voilà il y a un peu de tension làdessus.

L'auto-confrontation montre que le contrat didactique n'est pas encore établi avec cette classe du point de vue du professeur. Les échanges se déroulant en début d'année, l'enseignant veut montrer aux étudiants qu'il connaît les pratiques réelles, y compris les pratiques à la marge de la professionnalité officielle, que ce qui est « divulgué » en classe n'est pas à rapporter en entreprise, que les étudiants sont avant tout en situation d'apprendre, pas en situation de défendre ni de dénigrer l'entreprise qui les accueille. Le professeur se donne notamment pour objectif de créer un climat de confiance dans la classe, climat qui permet d'aborder les pratiques réelles dans une visée de formation, de compréhension, de comparaison des pratiques d'une entreprise à l'autre. Il a senti de la culpabilité de la part de l'étudiant «il se dit « je dénonce quelque chose ». Son intervention lors de l'échange semble chercher à déculpabiliser l'étudiant, notamment par une double stratégie. Premièrement en affirmant que cette pratique professionnelle, peu élégante, est largement répandue «c'est assez fréquent dans les entreprises ». Deuxièmement, en cherchant à l'expliquer, plutôt qu'à la dénoncer; l'enseignant renvoie à la logique économique de l'entreprise («tu sais combien ça coûte?»).

\section{4.- Les délais de séchage du ciment : légitimer de futurs enseignements}

Un autre étudiant explique qu'il a posé quelques kilomètres de clôture. L'enseignant l'interroge notamment sur les délais de séchage des poteaux de clôture sur lesquels vont être tendus des fils.

P: Il y en a pas mal là qui ont fait des clôtures là ; alors la question c'est dans les clôtures, il faut attendre combien de temps entre le moment où on met les poteaux et puis qu'on commence à mettre le fil de fer et le grillage: on peut faire ça le soir et recommencer le lendemain ou il faut attendre que ça sèche?

E8: Ah non, ah non, il faut attendre que ça sèche.

$\mathrm{P}$ : Combien de temps?

E8 : Normalement ben nous on laisse à peu près une semaine; mais normalement c'est 28 jours je crois qu'il faudrait pour que le béton a acquis sa capacité de résistance maximum

P: Et vous attendez 28 jours?

E8: On ben non, on va pas attendre 28 jours! on va pas perdre de temps non plus.

$\mathrm{P}$ : Alors combien de temps, concrètement.

E8: Là, ben une semaine, quatre cinq jours, parce qu'en général on tend pas le grillage tout de suite on met les...

E9 (Etudiant 9): Nous en général on attend 48 heures.

P: 48 heures

La classe : rire

E8 : Ben tu poses pas le grillage et tu tends au bout de 48 heures hein!

E9: Ben, souvent oui, parce que c'est des chantiers assez pressés.

E8: On a fait les pieux en béton [...] 2, 3 jours après on tend les câbles [...]

$\mathrm{P}$ : Est-ce qu'il y a moyen que le béton, d'augmenter le séchage du béton, que le béton durcisse plus vite?

E8: Un durcisseur

P: Vous connaissez ces produits?

E8: Non 
Lors des poses de clôtures, les délais de séchage de la partie maçonnée sont peu respectés. Les agents tendent des fils métalliques sur des poteaux dont le béton n'est pas suffisamment sec, ce qui nuit à la résistance des matériaux. Les risques de fissures et d'effondrements des clôtures peuvent être élevés. Le recours à des produits de type durcisseur permet de contourner le problème des délais de séchage.

On remarque que c'est le professeur qui introduit le sujet dans l'échange. Comme pour le cas de «l'enterrement civil », c'est l'enseignant qui incite à « faire avouer » des pratiques professionnelles qui s'écartent des règles de l'art. Il donne une solution technique, celle du durcisseur, pour pallier les inconvénients de cette pratique. Il ne développera pas cette solution, l'enjeu de la séance de retour de stage étant pour cet enseignant plus de créer des ruptures épistémiques que d'institutionnaliser des savoirs professionnels (Veyrac, 2006). La tâche appropriée de l'enseignant semble être proche de «faire avouer à l'étudiant des pratiques effectives ». Les enjeux de cette tâche sont multiples. Nous avons identifié celui relatif au contrat didactique qui apparaît dans l'entretien d'auto-confrontation et qui peut être résumé ainsi « vous devez parler des pratiques réelles, pas des pratiques idéalisées, pour qu'on puisse avoir un échange constructif pour votre formation ». Nous identifions ici un enjeu relatif aux aspects motivationnels de l'apprentissage: l'enseignant s'appuie sur des pratiques réelles discutées pour justifier l'enseignement technique qu'il proposera plus tard (le durcisseur). Les étudiants prennent conscience des limites de leurs connaissances (ils ne connaissent pas ce produit) et des enjeux de cette connaissance pour la pratique du métier. L'enjeu pour le professeur est de préparer les étudiants à être attentifs aux cours qui seront dispensés par la suite, lors de séances d'enseignement plus classiques.

\section{5.- Une tâche appropriée qui contribue à réduire le hiatus}

Dans le cas de l'appel d'offres, l'enseignant a été interpellé sur une pratique que l'étudiant jugeait peu conforme aux règlements. Dans le cas du port du casque, la pratique dangereuse est révélée par l'étudiant. L'enseignant joue de la provocation pour mettre en place une possibilité ultérieure de dialogue sans tabou sur ce thème. Pour les pratiques « d'enterrement civil » et de non-respect des délais de séchage, on a relevé une stratégie de «faire avouer » à l'étudiant des pratiques professionnelles non respectueuses des règles de l'art, pratiques déjà connues de l'enseignant.

Les conditions créées par cet enseignant lors de la séance de retour de stage semblent contribuer à réduire le hiatus entre l'institut de formation et le monde de l'entreprise. La tâche appropriée n'est pas de se donner les pratiques professionnelles comme référence à enseigner mais de créer un climat de confiance pour permettre à ces pratiques professionnelles d'être évoquées, analysées voire débattues. Ces évocations, analyses, débats légitiment des apports de connaissances à venir (réglementation de l'appel d'offres, formation à la santé sécurité au travail, durcisseur de béton, etc.) tout en augmentant probablement le sens des enseignements pour les étudiants et leur motivation dans la formation.

\section{5.- Le système des tâches appropriées}

\section{Trois tâches appropriées}

Cet article expose une démarche exploratoire qui vise à approcher le travail des enseignants, en privilégiant les points de vue des agents, au moyen de description de tâches appropriées.

Trois tâches appropriées ont été décrites. La première consiste à enseigner les pratiques professionnelles du référentiel tout en affichant aux apprenants l'existence d'un hiatus entre institut de formation et entreprise. La deuxième consiste à enseigner des pratiques professionnelles innovantes. Elle revient à déplacer le hiatus imposé par la prescription, mais sans nécessairement viser à le réduire. La troisième consiste à provoquer un discours des apprenants sur des pratiques professionnelles effec- 
tives, y compris des pratiques peu élégantes, en s'appuyant sur les séances de retour de stage. Cette approche compréhensive des pratiques effectives parvient probablement à réduire le hiatus institut de formation/entreprise, notamment en permettant à l'enseignant de justifier d'apports de contenus de cours.

Ces trois tâches relatent de la variabilité interindividuelle d'enseignants soumis à un ensemble de contraintes communes (même système de formation, même diplôme préparé, etc.).

\section{Nouvelles questions de recherche}

À l'issue de cette étude, il nous semble que plusieurs tâches appropriées relatives au hiatus entre travail en entreprise et formation peuvent coexister pour un même enseignant, dans un « système de tâches appropriées » en fonction de tel ou tel contenu à enseigner. C'est l'hypothèse que nous posons, à l'issue de cette approche exploratoire, et qui est schématisée par la figure 1.

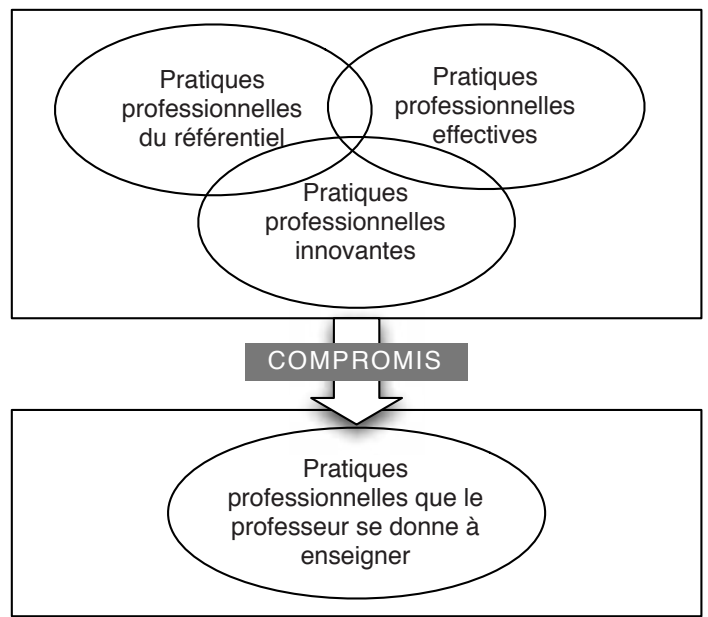

Figure 1: Composants du système de tâches appropriées de professeurs d'enseignement professionnel

Figure 1: System components of appropriate tasks of teachers from vocational education

La tâche appropriée des professeurs d'enseignement professionnel serait composée d'un ensemble de références à des pratiques professionnelles, pratiques pouvant s'opposer sur certains points. De cet ensemble parfois contrasté, un « compromis » s'opérerait; il en résulterait les pratiques professionnelles que le professeur se donne à enseigner.

Cette hypothèse est posée de manière relativement éloignée des propos de Vergnaud (2004), pour qui dans l'enseignement à visée professionnelle, « le modèle » de référence de l'enseignement, «c'est la pratique de la profession dans l'entreprise avec laquelle on travaille ». Cette assertion nous semble valide pour les formations où un seul style professionnel est visé par la formation, comme lors de formation en entreprise avec un tuteur. S'agissant d'un groupe d'apprenants, cet article montre une réalité plus complexe des pratiques professionnelles considérées comme modèle par les professeurs.

Le texte présent participe et contribue aux descriptions cliniques des modalités d'appropriation du prescrit mais reste une étude exploratoire. De plus, la question des liens entre difficultés des enseignants et tâche appropriée a été effleurée par les données mais reste à explorer. Les données ne permettent pas de faire correspondre tâches appropriées et difficultés à enseigner, voire souffrance au travail, mais ouvrent quelques perspectives dans ce sens. En effet, elles laissent voir que la tâche appropriée «assumer le hiatus en le posant comme un donné aux étudiants » est corrélée avec une faible motivation des apprenants, et de ce fait, des tensions à gérer dans la classe, voire des difficultés à enseigner.

Des recherches sur des effectifs plus importants d'enseignants sont nécessaires pour conforter les 
analyses avancées et prétendre dessiner les contours de la variabilité interindividuelle. Il est probable que les facteurs de cette variabilité soient multiples; citons l'expérience professionnelle de l'enseignant, ses liens avec les professionnels, les contenus à enseigner, le poids des prescriptions et les prises d'informations de l'enseignant (veille scientifique, visite de stage, échanges avec des collègues enseignants,...).

D'autres perspectives des recherches s'ouvrent. Par exemple, la prise en compte du point de vue des étudiants serait également à considérer pour aider les enseignants à adapter leurs tâches appropriées aux besoins réels des apprenants. Cette prise en compte est complexe, à la hauteur de la complexité de l'analyse du travail des enseignants. Le travail des enseignants étant multi-adressé (plusieurs apprenants dans une classe), les critères de performance sont difficiles à fixer. De plus, pour un même apprenant, les critères attendus concernant le travail d'un professeur sont multiples: « apprendre des gestes professionnels », « devenir citoyen », «passer une heure de cours agréable », « obtenir une bonne note à l'examen terminal », etc.

Des analyses plurielles illustrent la complexité de l'analyse du travail et la multiplicité des regards possibles. Par exemple, un seul cours d'un professeur d'école donne lieu à des analyses plurielles d'une dizaine de chercheurs (Vinatier, \& Altet, 2008). Malgré cette complexité, l'analyse du travail des enseignants se développe, probablement du fait des enjeux associés en terme notamment de formation des enseignants.

\section{RÉFÉRENCES}

Amigues, R. (2002). L'enseignement comme travail. In P. Bressoux (Ed.). Les stratégies d'enseignement en situation d'interaction (pp. 243-262). Note de synthèse pour Cognitique: Programme Ecole et Sciences Cognitives.

Anderson, J.R., \& Fincham, J.M. (1994). Acquisition of procedural skills from examples. Journal of Experimental Psychology: Learning, Memory and Cognition, 20, 1322-1340.

Asloum, N., \& Bouillier-Oudot, M.-H. (2007). Pratiques collectives d'enseignants engagés dans une formation interdisciplinaire. Congrès international Actualité et de la recherche en éducation en formation, Strasbourg. http://www.congresintaref.org/actes_pdf/AREF2007

Bardin, L. (1977). L'analyse de contenu. Paris: Presses Universitaires de France.

Bouillier, M.-H. (2006). Constructions de curriculums et définition de nouveaux métiers. In Y. Lenoir, \& M.H. Bouillier-Oudot (Eds.), Savoirs professionnels et curriculum de formation. Une variété de situations, une variété de conceptions, une variété de propositions (pp. 345-364). Québec: Presses de l'Université Laval.

Bouillier-Oudot, M.-H., \& Asloum, N. (2004). L'interdisciplinarité dans les formations à visée professionnelle. 14ème congrès international de l'Association Mondiale des Sciences de l'Education. Santiago du Chili, mai.

Bouillier, M.-H., Asloum, N., \& Veyrac, H. (2008). A quels modèles d'apprentissage se réfèrent les enseignants dans des formations à visée professionnelle? Une étude de cas dans l'enseignement technique agricole. In Y. Lenoir, \& P. Pastré (Eds.), Didactique professionnelle et didactique des disciplines en débat (pp. 189-211). Toulouse: Octarès Editions.

Caroly, S., \& Weill-Fassina, A. (2004). Evolutions des régulations de situations critiques au cours de la vie professionnelle dans des activités de relations de service. Le Travail Humain, 67, 305-332.

Delgoulet, C. (2001). La construction des liens entre situations de travail et situations d'apprentissage dans la formation professionnelle. Pistes, 3 (2), 1-12. http://www.pistes.uqam.ca/v3n2/articles/v3n2a2.htm

Falzon, P. (2004). Nature, Objectifs et connaissances de l'ergonomie, In P. Falzon (Ed.), Ergonomie (pp. 1735). Paris: Presses Universitaires de France.

Goigoux, R. (2002). Analyser l'activité d'enseignement de la lecture: une monographie. Revue Française de Pédagogie, 138, 125-134. 
Kovess-Masféty, V., Seidel, C., \& Sévilla, C. (2006). Difficulté au travail, souffrance au travail, médicalisation. Séminaire sur le travail enseignant. http://ep.inrp.fr/EP/formations/continue/seminaire travail enseignant/communication audio viviane kovess/view

Lantheaume, F., \& Hélou, C. (2008). La souffrance des enseignants, Une sociologie pragmatique du travail enseignant. Paris: Presses Universitaires de France.

Leblanc, S., Ria, L., Dieumegard, G., Serres, G., \& Durand, M. (2008). Concevoir des dispositifs de formation professionnelle des enseignants à partir de l'analyse de l'activité dans une approche enactive, Activités, 5 (1), 58-78. http://www.activites.org/v5n1/v5n1.pdf

Leplat, J. (1997). Regards sur l'activité en situation de travail Contribution à la psychologie ergonomique. Paris: Presses Universitaires de France.

Litim, M., Prot, B., Roger, J.-L., Ruelland, D., \& Clot, Y. (2005). Enjeux du travail et "genres" professionnels dans la recomposition en cours des métiers de la fonction publique. Le cas des professeurs de l'enseignement secondaire et du personnel soignant de gérontologie. Rapport de recherche, Paris: Laboratoire de psychologie du travail et de l'action, CNAM.

Raisky, Cl. (1993). Problème du sens des savoirs professionnels préalables à une didactique. In P. Jonnaert, \& Y. Lenoir (Eds.), Sens des didactiques et didactiques du sens (pp. 101-122). Sherbrooke: Edition du CRP.

Rey, B. (2006). Compétences professionnelles et curriculum: des réalités conciliables? In Y. Lenoir, \& M-H. Bouillier-Oudot (Eds.), Savoirs professionnels et curriculum de formation. Une variété de situations, une variété de conceptions, une variété de propositions (pp.83-107). Québec: Presses de l'Université Laval.

Rogalski, J. (2003). Y a-t-il un pilote dans la classe? Une analyse de l'activité de l'enseignant comme gestion d'un environnement dynamique ouvert. Recherches en Didactique des Mathématiques, 23, 343-388.

Salembier, P., Kahn, J., Calvet, G., Zouinar, M., \& Relieu, M. (2005). "Just Follow Me" - Examining the Use of a Multimodal Mobile Device in Natural Settings. In G. Salvendy (Ed.), 11 th International Conference on Human-Computer Interaction, LEA.

Samurçay, R., Rabardel, P. (2004). Modèles pour l'analyse de l'activité et des compétences, propositions. In R. Samurçay, \& P. Pastré (Eds.), Recherches en didactique professionnelle (pp. 163-180). Toulouse: Octares.

Tardif, M., Lessard, C. (1999). Le travail enseignant au quotidien. Expérience, interactions humaines et dilemmes professionnels, Québec: Presses de l'Université Laval et Bruxelles, De Boeck.

Tochon, F.V. (1993). L'enseignant expert. Paris : Nathan.

Vergnaud, G. (2004). Les archives audiovisuelles de la recherche en sciences humaines et sociales, chapitre «Didactique professionnelle et didactique des disciplines » http://semioweb.msh-paris.fr/AAR/413/ introduction. $\operatorname{asp}$ ? id=413

Vermersch, P. (1985). Données d'observation sur l'utilisation d'une consigne écrite: l'atomisation de l'action, Travail Humain, 48, 161-172.

Veyrac, H. (2006). Analyse d'une activité d'explicitation des expériences préprofessionnelles des élèves en classe. Biennale de l'éducation, Expérience(s), savoir(s), sujet(s), Lyon, France, 11-14 avril http://www. inrp.fr/biennale/8biennale/contrib/

Veyrac, H., \& Chatigny, C. (2007). Approche de la réussite scolaire par l'analyse ergonomique du travail des enseignants intervenant auprès d'élèves en formation professionnelle, Colloque international Recherche en Education et en Formation, Apprendre et former : pour quelles réussites scolaires?, Université de Sherbrooke, Octobre.

Vinatier, I., \& Altet, M. (Eds.) (2008). Analyser et comprendre la pratique enseignante. Rennes: Presses Universitaries de rennes.

Woods, P. (1977/1997). Les stratégies de « survie» des enseignants. In J.-C. Forquin (Ed.), Les sociologues de l'éducation américains et britanniques, Présentation et choix de textes (pp. 351-376). Paris: De Boeck Université, INRP. 


\section{RÉSUMÉ}

Les enseignants de disciplines professionnelles sont confrontés à un ensemble de prescriptions spécifiques du fait de la visée professionnelle des savoirs à enseigner. Ces savoirs sont prescrits par les référentiels. La mise en mot imposée par l'écriture des référentiels a un effet réducteur de l'activité professionnelle visée par les formations. Elle est une des causes du hiatus entre formation et travail. Les enseignants perçoivent-ils ce hiatus? Quelles tâches se donnent-t-ils à réaliser à son propos? Des réponses sont apportées par la description de tâches appropriées. Des entretiens de recherche, observations de séance de cours et autoconfrontations dans le secteur de l'enseignement en aménagement paysager permettent de mieux comprendre le travail des enseignants de filières professionnelles. Les données illustrent différentes tâches appropriées de professeurs: assumer le hiatus en l'annonçant comme un donné aux apprenants, enseigner des pratiques innovantes, faire apparaître les contradictions entre pratiques du référentiel et pratiques professionnelles effectives. Cet article illustre une réduction du hiatus et contribue au développement des recherches sur l'analyse du travail des enseignants.

\section{MoTS CLÉS}

Tâche appropriée, prescription, pédagogie de l'alternance, enseignement professionnel, travail enseignant

\section{RESUMEN}

Las tareas apropiadas de los docentes de las disciplinas profesionales. Ilustración de la distancia que existe entre la formación y el mundo del trabajo. Los docentes de las disciplinas profesionales de la educación secundaria deben hacer frente a numerosas dificultades específicas a la orientación profesional de los contenidos de sus materias. Estos contenidos son establecidos en documentos oficiales. La puesta en palabras impuesta por la escritura de estos documentos tiene un efecto reductor de la actividad profesional objeto de la formación, reducción que produce un aumento de la distancia que existe entre formación y el mundo del trabajo. ¿Perciben los docentes esta distancia? ¿Cómo intentan disminuirla? En este artículo, intentaremos dar una respuesta a estas interrogaciones a través de la descripción de "tareas apropiadas". Para comprender mejor el trabajo de los docentes de la educación secundaria profesional, nos basaremos en entrevistas, observaciones de clases, y auto-confrontaciones, concentrándonos en el sector de la enseñanza en Paisajismo. Los datos ilustran diferentes tareas apropiadas de docentes: asumir la distancia escuela / empresa anunciándola a los alumnos como un simple dato de la realidad, enseñar prácticas innovadoras, poner en evidencia las contradicciones entre los programas oficiales y las prácticas profesionales reales. Este artículo ilustra una reducción de la distancia entre el sistema educativo y el mundo profesional, y contribuye asimismo al desarrollo de la investigación sobre el análisis del trabajo de los docentes.

\section{PALABRAS CLAVE}

Tarea apropiada, prescripción, pedagogía de la formación en alternancia, educación profesional, trabajo del docente 


\section{RÉFÉRENCEMENT}

Veyrac, H. \& Asloum, N. (2009). Les tâches appropriées des professeurs d'enseignement professionnel. Illustration du hiatus entre travail en entreprise et formation. Activités, 6 (1), pp. 69-86. http://www. activites.org/v6n1/v6n1.pdf

Article soumis le 1er avril 2008 accepté pour publication le 13 mars 2009 